\title{
Surface plasmon coupling effect in an InGaN/GaN single-quantum-well light-emitting diode
}

\author{
Dong-Ming Yeh, Chi-Feng Huang, Cheng-Yen Chen, Yen-Cheng Lu, and C. C. Yang ${ }^{\text {a) }}$ \\ Institute of Photonics and Optoelectronics and Department of Electrical Engineering, National \\ Taiwan University, 1, Roosevelt Road, Section 4, Taipei, 106 Taiwan, Republic of China
}

(Received 8 September 2007; accepted 4 October 2007; published online 23 October 2007)

\begin{abstract}
The authors demonstrate the coupling effects between the quantum well (QW) and surface plasmon (SP) generated nearby on the $p$-type side in an InGaN/GaN single-QW light-emitting diode (LED). The QW-SP coupling leads to the enhancement of the electroluminescence (EL) intensity in the LED sample designed for QW-SP coupling and reduced SP energy leakage, when compared to a LED sample of weak QW-SP coupling or significant SP energy loss. In the LED samples of significant QW-SP coupling, the blueshifts of the photoluminescence and EL emission spectra are observed, indicating one of the important features of such a coupling process. The device performance can be improved by using the $n$-type side for SP generation such that the device resistance can be reduced and the QW-SP coupling effect can be enhanced (by further decreasing the distance between the QW and metal) because of the higher carrier concentration in the $n$-type layer. (C) 2007 American Institute of Physics. [DOI: 10.1063/1.2802067]
\end{abstract}

The coupling between a semiconductor quantum well (QW) and a surface plasmon (SP) can create an alternative channel of light emission besides that directly through carrier recombination. In this channel, the energy in carriers is first transferred into the SP modes, which are induced by the QW spontaneous emission on a metal structure nearby, through a process similar to stimulated emission. The SP modes can then be coupled into radiation mode if the momentum matching condition is satisfied. Such a photon emission channel can be particularly effective when the SP energy loss is relatively lower than that through the nonradiative carrier recombination. In this situation, the spontaneous emission rate and the light extraction efficiency of a light-emitting device can be simultaneously enhanced. Although the coupling between an InGaN/GaN QW and SPs for light emission enhancement has been widely studied, ${ }^{1-9}$ so far only the photoluminescence (PL) measurement was demonstrated. For practical application of the QW-SP coupling, the enhancement of electroluminescence (EL) in a light-emitting diode (LED) needs to be illustrated.

In this letter, we demonstrate the variations of EL spectrum and intensity in an InGaN/GaN single-QW LED due to the QW-SP coupling by comparing three LED samples of different device structures. The LED sample of significant QW-SP coupling and low SP energy loss shows the enhancements of EL intensity and the significant blueshifts of emission spectra in the PL and EL measurements when compared with a standard and a flip-chip LED samples. The amount of EL enhancement is quite consistent with what was previously predicted. ${ }^{3}$ The QW-SP coupling is expected to also occur in the flip-chip LED. The epitaxial structure was grown on $c$-plane sapphire substrate with metal organic chemical vapor deposition. It consists of a $25 \mathrm{~nm}$ nucleation layer, a $2 \mu \mathrm{m} n$-type GaN layer, a single $\mathrm{InGaN} / \mathrm{GaN} \mathrm{QW}$ structure $(3 \mathrm{~nm}$ in well width and $8 \mathrm{~nm}$ in the widths of the upper and lower undoped $\mathrm{GaN}$ barrier layers), a $10 \mathrm{~nm}$

a) Tel.: 886-2-23657624. FAX: 886-2-23652637. Electronic mail: ccy@cc.ee.ntu.edu.tw p-type $\mathrm{Al}_{0.2} \mathrm{Ga}_{0.8} \mathrm{~N}$ current blocking layer, and finally a $70 \mathrm{~nm} p$-type $\mathrm{GaN}$ layer. The internal quantum efficiency of the QW was estimated to be $40 \%$ based on temperaturedependent PL measurement.

As shown in Fig. 1 for the structure of the SP-coupled LED (referred to as LED III), in which the QW-SP coupling is expected to occur, a periodical strip pattern of $\mathrm{Ni}$ $(4 \mathrm{~nm}) / \mathrm{Au}(3 \mathrm{~nm})$ was coated with electron-gun evaporation (then annealed at $550{ }^{\circ} \mathrm{C}$ for $30 \mathrm{~s}$ in ambient $\mathrm{N}_{2}$ ) on the mesa surface, except the region of the $p$-contact pad, with the strip width at $10 \mu \mathrm{m}$ and spacing also at $10 \mu \mathrm{m}$ for current spreading [referred to as the current spreading layer, (CSL), in Fig. 1]. The $p$ contact consists of the coatings of $\mathrm{Ni}$ $(15 \mathrm{~nm}) / \mathrm{Au}(150 \mathrm{~nm})$, below which a $40 \mathrm{~nm} \mathrm{SiO} \mathrm{Si}_{2}$ dielectric layer was deposited with the plasma enhanced chemical vapor deposition to enhance lateral current spreading. After the coatings of $\mathrm{Ti} \quad(15 \mathrm{~nm}) / \mathrm{Al} \quad(75 \mathrm{~nm}) / \mathrm{Ti} \quad(15 \mathrm{~nm}) / \mathrm{Au}$ $(150 \mathrm{~nm})$ for the $n$-type Ohmic contact, the standard LED sample (referred to as LED I) is completed. In the flip-chip LED (referred to as LED II), a $50 \mathrm{~nm} \mathrm{Ag} \mathrm{film} \mathrm{was} \mathrm{deposited}$ on the current-spreading strip structure such that top emis-

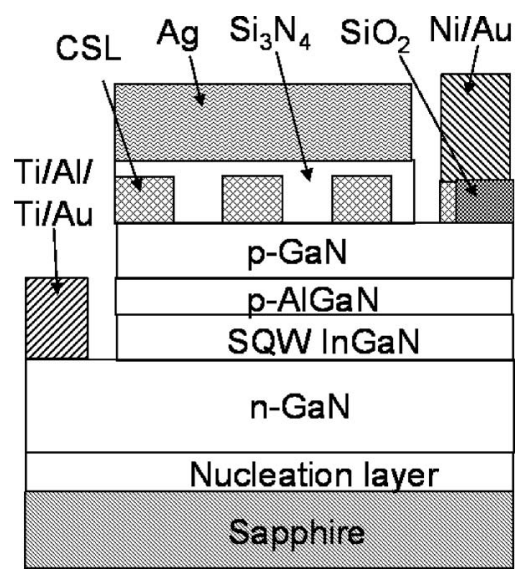

FIG. 1. Device structure of the LED with SP coupling (LED III). Current spreading layer. 


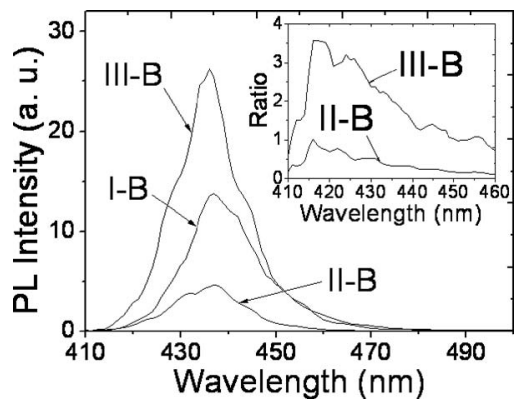

FIG. 2. PL spectra of the three samples with bottom excitation and bottom detection. The inset shows the PL intensity ratio using the standard LED (LED I-B) as the reference.

sion is suppressed. This Ag film serves to generate SPs at the $\mathrm{Ag} / \mathrm{GaN}$ interface for QW-SP coupling. As shown in Fig. 1 for LED III, to reduce SP leakage through the direct metal/ semiconductor contact in the SP-coupled LED, ${ }^{9}$ a $10 \mathrm{~nm}$ $\mathrm{Si}_{3} \mathrm{~N}_{4}$ dielectric layer was deposited to cover the $\mathrm{Ni} / \mathrm{Au}$ current-spreading strip structure before the final coating of a $50 \mathrm{~nm} \mathrm{Ag}$ thin film on the top for generating SPs. Therefore, the SP-generating $\mathrm{Ag}$ film is isolated from the $\mathrm{Ni} / \mathrm{Au}$ current-spreading strip structure and the $p$ contact.

In the EL measurements, the output spectra and intensities from both the top and bottom (sapphire substrate) sides are recorded. The outputs from the top and bottom sides are denoted as "-T" and "-B," respectively. Figure 2 shows the PL spectra of the three LED samples indicating their relative intensities. The PL measurements were performed with the configuration of bottom excitation and bottom detection. Although the strong reflection in the configuration of full metal coverage on the top sides of LEDs II and III is supposed to result in higher PL intensities in these two samples, the relatively weaker PL intensity in LED II shown in Fig. 2 implies the occurrence of QW-SP coupling in this sample. The reduction of PL intensity is due to the SP energy leakage through the metal/semiconductor interface after the energy of carriers is transferred into SP modes. The QW-SP coupling is expected to also occur in LED III because of their similar structures. In this sample, because of the shield of the $\mathrm{Si}_{3} \mathrm{~N}_{4}$ dielectric layer, the SP loss is reduced and hence the emission enhancement through QW-SP coupling is observed. The other feature proving the occurrence of QW-SP coupling in Fig. 2 is the blueshifts of PL spectra in LEDs II and III. ${ }^{10}$ The center-of-mass energy of the PL spectrum is blueshifted from $2.817 \mathrm{eV}$ in LED I to 2.841 and $2.838 \mathrm{eV}$ in LEDs II and III, respectively. Such blueshifts are clearly manifested in the ratios of PL intensity by using that of LED I for normalization, as shown in the insert of Fig. 2.

Figure 3 shows the EL spectra of the six outputs of the three samples at the injection current of $20 \mathrm{~mA}$ indicating their relative intensities. Here, one can see the strongest and weakest EL intensities from LEDs III and II, respectively, in the comparison of bottom emission. Also, the strongest and weakest intensities from LEDs I and II, respectively, in the comparison of top emission can be observed. The significantly higher intensities of LEDs III-B and III-T in comparing with those of LEDs II-B and II-T, respectively, are attributed to the QW-SP coupling in these two samples and the significant SP leakage in LED II. The QW-SP coupling in LED I is expected to be weak because the strip coating of $\mathrm{Ni} / \mathrm{Au}$ for the CSL is ineffective for SP generation in the Downloaded 20 Feb 2009 to 140.112 .113 .225 . Redistribution subje

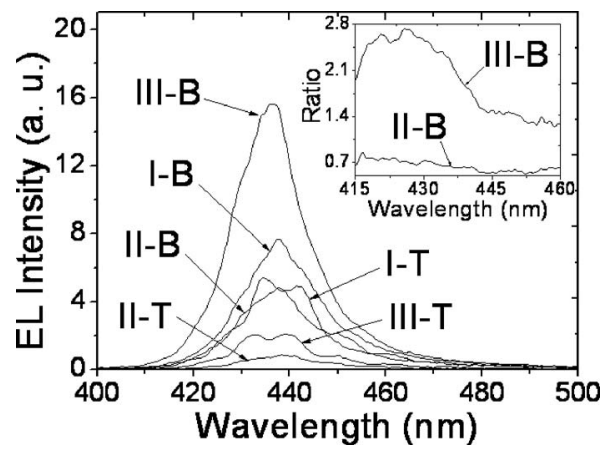

FIG. 3. EL spectra of the six emission outputs from the three samples when the injection current is $20 \mathrm{~mA}$. The inset shows the EL intensity ratio using the bottom-emitting standard LED output (LED I-B) as the reference.

QW-emitting wavelength range. Just like the PL data, the EL spectra in Fig. 3 also show blueshifts due to the QW-SP coupling in LEDs II and III, particularly for the bottom emission. The EL spectral center-of-mass energy of bottom emission is blueshifted from $2.815 \mathrm{eV}$ in LED I-B to 2.819 and $2.833 \mathrm{eV}$ in LEDs II-B and III-B, respectively. The blueshifts are clearly shown in the EL intensity ratios, as shown in the insert of Fig. 3. The blueshift trend of top emission is less prominent. The EL spectral center-of-mass energy of top emission is shifted from $2.814 \mathrm{eV}$ in LED I-T to 2.812 and $2.821 \mathrm{eV}$ in LEDs II-T and III-T, respectively. The difference between the bottom and top emitting outputs can be attributed to the metal absorption and a more complicated SP coupling process (involved in the SP modes at the air/metal interface), which affect the top emission spectra. ${ }^{11}$

Figure 4 shows the EL intensities of the six outputs as functions of injection current. The relative intensities of the six outputs are unchanged in increasing the injection current. Figure 5 shows the variation of the summation of top and bottom-emitting intensities in each sample with injection current. Although such a summation does not represent the total emission power from a sample, it can indicate the relative output intensity of an LED sample assuming that the radiation patterns of the three samples are similar. In Fig. 5, one can see the enhancement and reduction of the output intensities from LEDs III and II, respectively, when compared with that of LED I. By comparing the results of LEDs I and III, one can see that the QW-SP coupling leads to $25 \%-50 \%$ output enhancement.

The injection currents used in Figs. 4 and 5 are relatively small when compared with that for a conventional LED due to the large device resistance in the LEDs under study, as

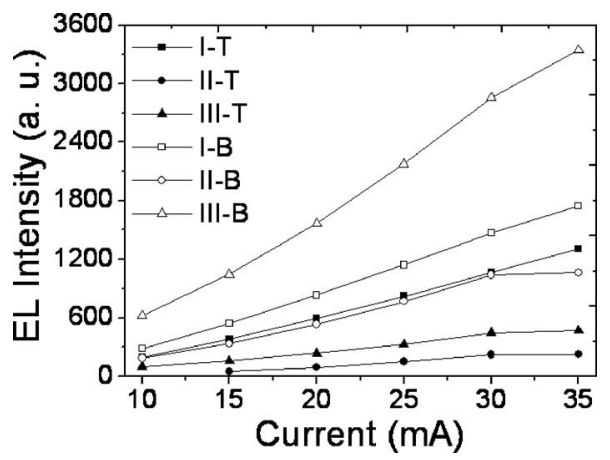

FIG. 4. LED intensities of the six emission outputs from the three samples as functions of injection current.

to AIP license or copyright; see http://apl.aip.org/apl/copyright.jsp 


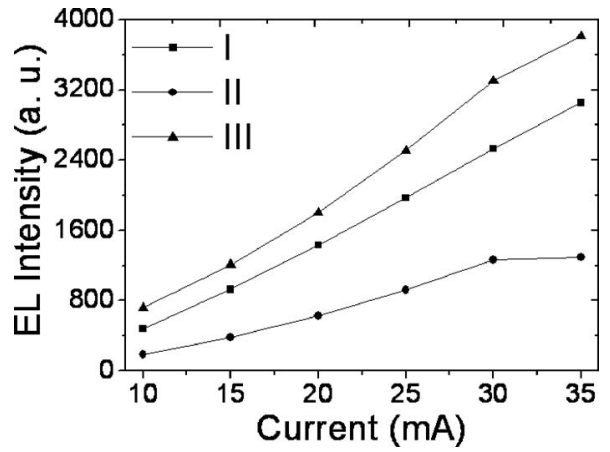

FIG. 5. Summations of the top and bottom-emitting intensities of the three samples as functions of injection current.

shown in the $I-V$ curves of Fig. 6. Here, one can see that although the turn-on voltages of the three samples are all around $3 \mathrm{~V}$, their $20 \mathrm{~mA}$ voltages are larger than $6 \mathrm{~V}$. However, the almost zero current level $(<\sim 1 \mu \mathrm{A})$ in the reverse-biased voltage range indicates that the leakage currents of the three samples are low. The high resistances of those devices can be due to the thin $p$-type layer, which normally has a thickness of $>200 \mathrm{~nm}$ in a conventional LED, and hence has poor current spreading. The device resistance can be reduced by increasing the thickness of the $p$-type GaN layer and using the $n$-type side for SP coupling in a vertical LED structure. ${ }^{12,13}$ Because normally the electron concentration in an $n$-type $\mathrm{GaN}$ layer can be quite high (up to $10^{20} \mathrm{~cm}^{-3}$ ) and hence current spreading can be quite

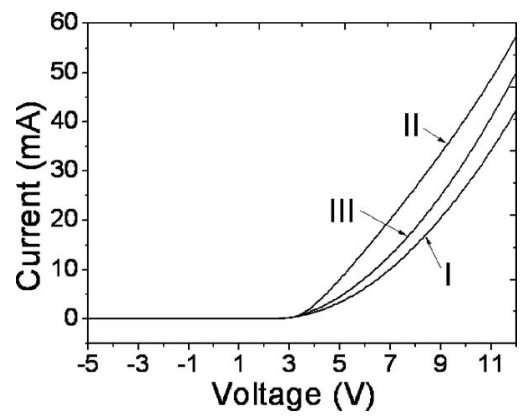

FIG. 6. Current vs voltage ( $I-V$ curves) of the three samples. effective even with a thin $n$-type layer, the device resistance will not be sacrificed in reducing the $n$-type GaN layer down to a few tens of nanometer. In this situation, a thinner $n$-type layer can lead to stronger QW-SP coupling and higher EL output enhancement.

In summary, we have demonstrated the QW-SP coupling effects in an InGaN/GaN single-QW LED. The QW-SP coupling led to the enhancement of the EL intensity in the LED sample designed for the coupling and reduced SP energy leakage, when compared with the LED sample of weak QW-SP coupling or significant SP energy loss. In the LED samples of significant QW-SP coupling, the blue shifts of the PL and EL emission spectra were observed.

This research was supported by National Science Council, The Republic of China, under Grant Nos. NSC 96-2120M-002-008 and NSC 96-2628-E-002-044-MY3, and by U.S. Air Force Scientific Research Office under Contract No. AOARD-07-4010.

${ }^{1}$ K. Okamoto, I. Niki, A. Shvartser, Y. Narukawa, T. Mukai, and A. Scherer, Nat. Mater. 3, 601 (2004).

${ }^{2}$ K. Okamoto, I. Niki, A. Scherer, Y. Narukawa, T. Mukai, and Y. Kawakami, Appl. Phys. Lett. 87, 071102 (2005).

${ }^{3}$ G. Sun, J. B. Khurgin, and R. A. Soref, Appl. Phys. Lett. 90, 111107 (2007).

${ }^{4}$ J. B. Khurgin, G. Sun, and R. A. Soref, J. Opt. Soc. Am. B 24, 1968 (2007).

${ }^{5}$ C. Y. Chen, D. M. Yen, Y. C. Lu, and C. C. Yang, Appl. Phys. Lett. 89, 203113 (2006).

${ }^{6}$ C. Y. Chen, Y. C. Lu, D. M. Yeh, and C. C. Yang, Appl. Phys. Lett. 90, 183114 (2007).

${ }^{7}$ Y. C. Lu, C. Y. Chen, D. M. Yeh, C. F. Huang, T. Y. Tang, J. J. Huang, and C. C. Yang, Appl. Phys. Lett. 90, 193103 (2007).

${ }^{8}$ D. M. Yeh, C. Y. Chen, Y. C. Lu, C. F. Huang, and C. C. Yang, Nanotechnology 18, 265402 (2007).

${ }^{9}$ D. M. Yeh, C. F. Huang, Y. C. Lu, C. Y. Chen, T. Y. Tang, J. J. Huang, K. C. Shen, Y. J. Yang, and C. C. Yang, Appl. Phys. Lett. 91, 063121 (2007).

${ }^{10}$ A. Neogi, C. W. Lee, H. O. Everitt, T. Kuroda, A. Tackeuchi, and E. Yablonvitch, Phys. Rev. B 66, 153305 (2002).

${ }^{11}$ J. Feng, T. Okamoto, and S. Kawata, Opt. Lett. 30, 2302 (2005).

${ }^{12}$ H. Kim, K. K. Kim, K. K. Choi, H. Kim, J. O. Song, J. Cho, K. H. Baik, C. Sone, Y. Park, and T. Y. Seong, Appl. Phys. Lett. 91, 023510 (2007).

${ }^{13}$ O. B. Shchekin, J. E. Epler, T. A. Trottier, T. Margalith, D. A. Steigerwald, M. O. Holcomb, P. S. Martin, and M. R. Krames, Appl. Phys. Lett. 89, 071109 (2006). 